\title{
Research of DOA Estimation Based on Modified MUSIC Algorithms Liwei HUANG ${ }^{1, a}$ Yulin $\mathrm{CHEN}^{2, b}$ Huiqin $\mathrm{CHEN}^{3, \mathrm{c}}$ \\ ${ }^{1,2,3}$ Department of Electrical Engineering , Chengxian college of Southeast University, Nanjing, 210088, China \\ aemail:liwei8203@163.com, bemail: 4817382@qq.com, cemail: 41024257@qq.com
}

Keywords: Root-MUSIC;Beamforming MUSIC;Forward/Backward Spatial Smoothing MUSIC

Abstract: This paper studys several kinds of MUSIC algorithms in DOA estimation, expounds the principle of Root-MUSIC algorithm, beamforming MUSIC algorithm, spatial smoothing MUSIC algorithm, conductes simulation for uniform linear array with Matlab, get the characteristics of three kinds of algorithms, Root-MUSIC algorithm is suitable for high SNR, the performance of beamforming MUSIC depends on the selection of matrix, spatial smoothing MUSIC gets the DOA estimation of coherent signals with the cost of reducing the antenna aperture, provides a reference for practical use.

\section{Introduction}

DOA estimation is an important part of array signal processing. The higher the accuracy of DOA estimation, the stronger the inhibition of interference. After the classical MUSIC algorithm, there are a variety of modified MUSIC algorithms. This paper compares the characteristics of Root-MUSIC, beamforming MUSIC and forward/backward spatial smoothing MUSIC, simulates the algorithms with Matlab in different conditions, provides a reference for practical use.

\section{Root-MUSIC}

Define a polynomial

$$
f(z)=e^{H}{ }_{i} p(z) \quad i=N+1, \ldots M
$$

$e_{i}$ is the eigenvector corresponding to the small eigenvalues of data covariance matrix, $p(z)=\left[\begin{array}{lll}1 & z & \cdots \\ z^{M-1}\end{array}\right]^{T}$. When $z=e^{j \omega}$, the roots of polynomial are on unit circle, $p\left(e^{j \omega}\right)$ is the direction vector of frequency $\omega$. The vector which is quadrature to the noise subspace is the direction vector of signal, so the polynomial can be modified to

$$
f(z)=p^{H}(z) U_{N} U^{H}{ }_{N} p(z) \text {. }
$$

Searching the polynomial's roots to find out the root on the unit circle, using formula (3)to find the corresponding source Angle ${ }^{[1]}$.

$$
\theta_{i}=\arcsin \left(\frac{\lambda}{2 \pi d} \arg \left\{\hat{z}_{i}\right\}\right)
$$

Experiment uses 3 separate narrowband signal source, the center frequency is $1000 \mathrm{~Hz}$, incident to the linear array from 60 degrees, 30 degrees, 75 degrees, the sampling number is 128, take half wavelength as array element spacing. Take different signal-to-noise ratio for simulation, the results are shown in figure 1. 


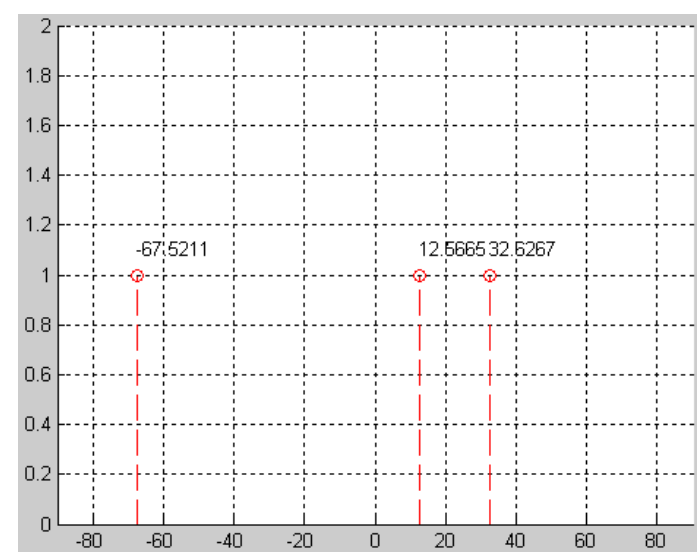

(a) SNR is -20

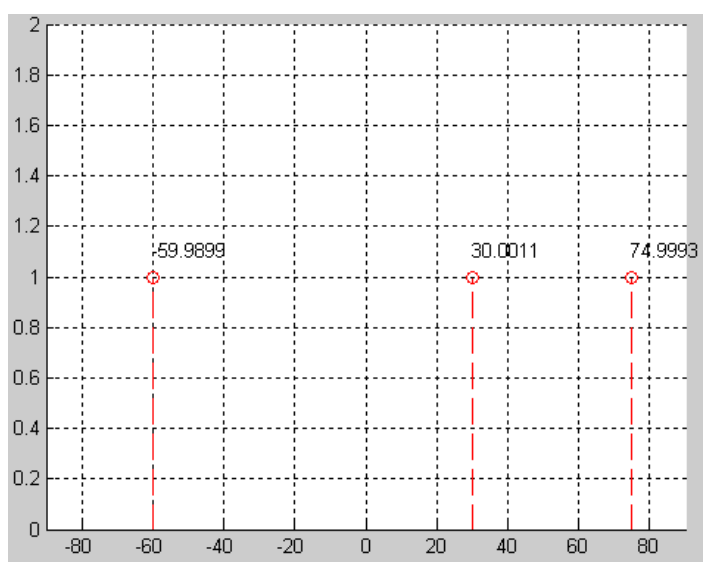

(c) SNR is 10

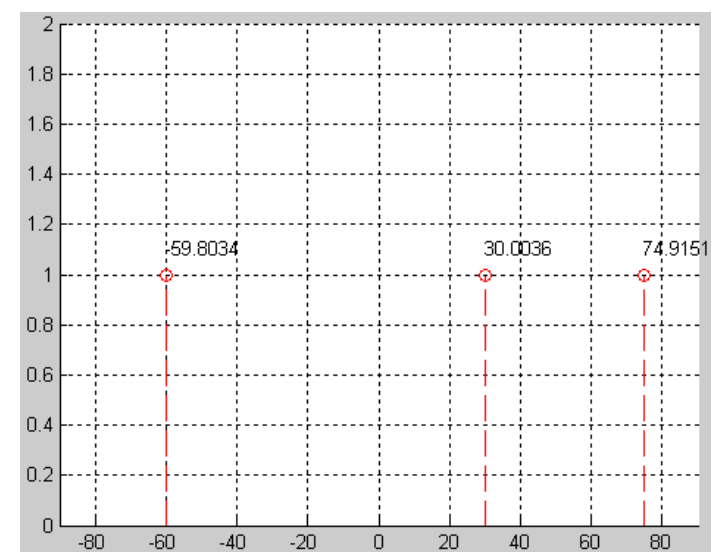

(b) SNR is -5

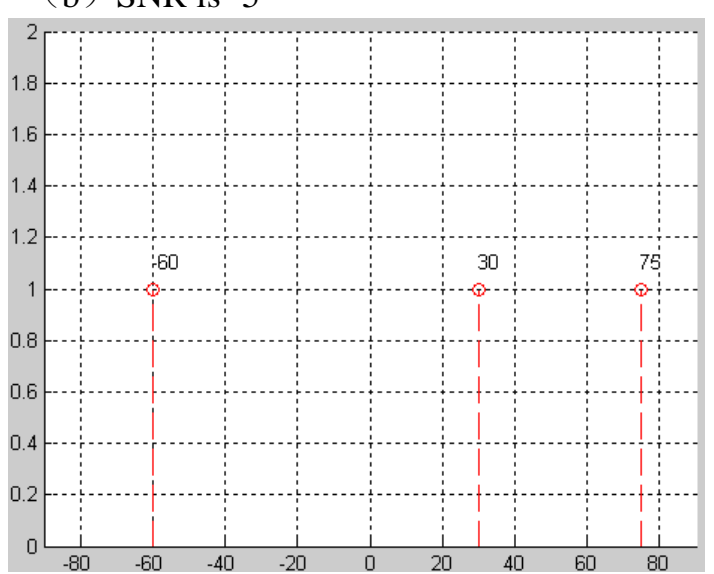

(d) SNR is 30

Fig.1. Estimation performance of different signal-to-noise ratio with Root-MUSIC

Figure 1 shows that perfomence of Root-MUSIC is poor when there is low signal-to-noise ratio, with the increase of signal-to-noise ratio, the resolution of MUSIC algorithm for DOA estimation has improved ${ }^{[2]}$.

\section{Beamforming MUSIC}

Each array element of Classical MUSIC algorithm corresponds to a data processing channel, Beamforming MUSIC transforms the arrays to several beams, uses the beam data to estimate DOA. The principle is shown in figure 2. The method can be summarized as the following steps: use the array of receiving data to get data covariance matrix, choose different beamformings to form matrix, use the data matrix of beam space to estimate spectrum, find signal direction of the corresponding maximum ${ }^{[3]}$.

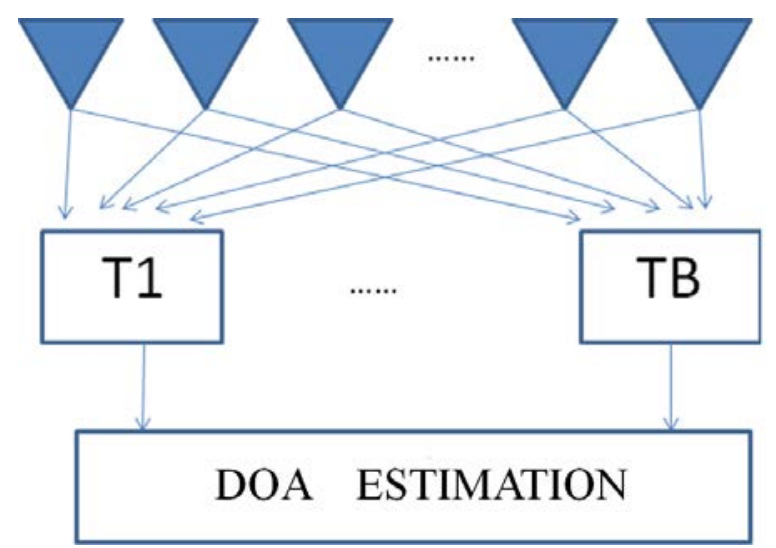

Fig.2. Schematic diagram of beamforming MUSIC

Experiment uses 10 array elements, 3 separate narrowband signal source, the center frequency is $1000 \mathrm{~Hz}$, signal-to-noise ratio is $10 \mathrm{~dB}$, the sampling number is 128 , array element spacing is half wavelength. Take different directions to incident to the linear array for simulation, the results are 
shown in figure 3.
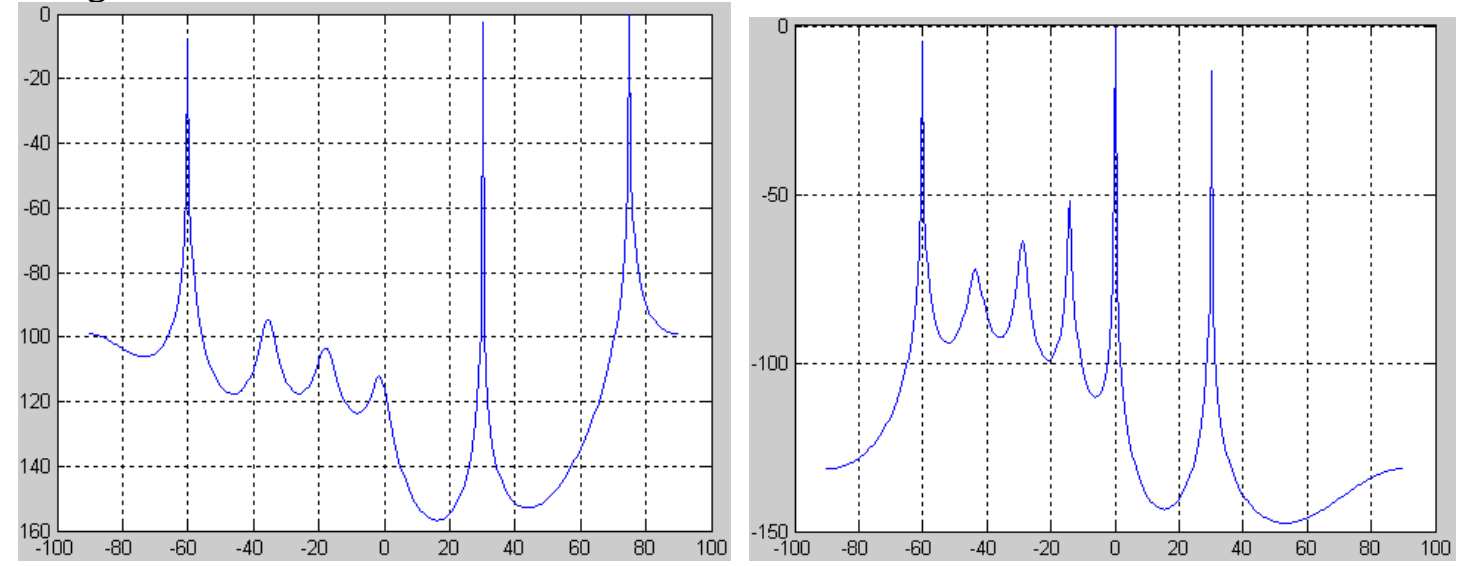

(a) incident direction:-60,30,75

(b) incident direction: $-60,0,30$
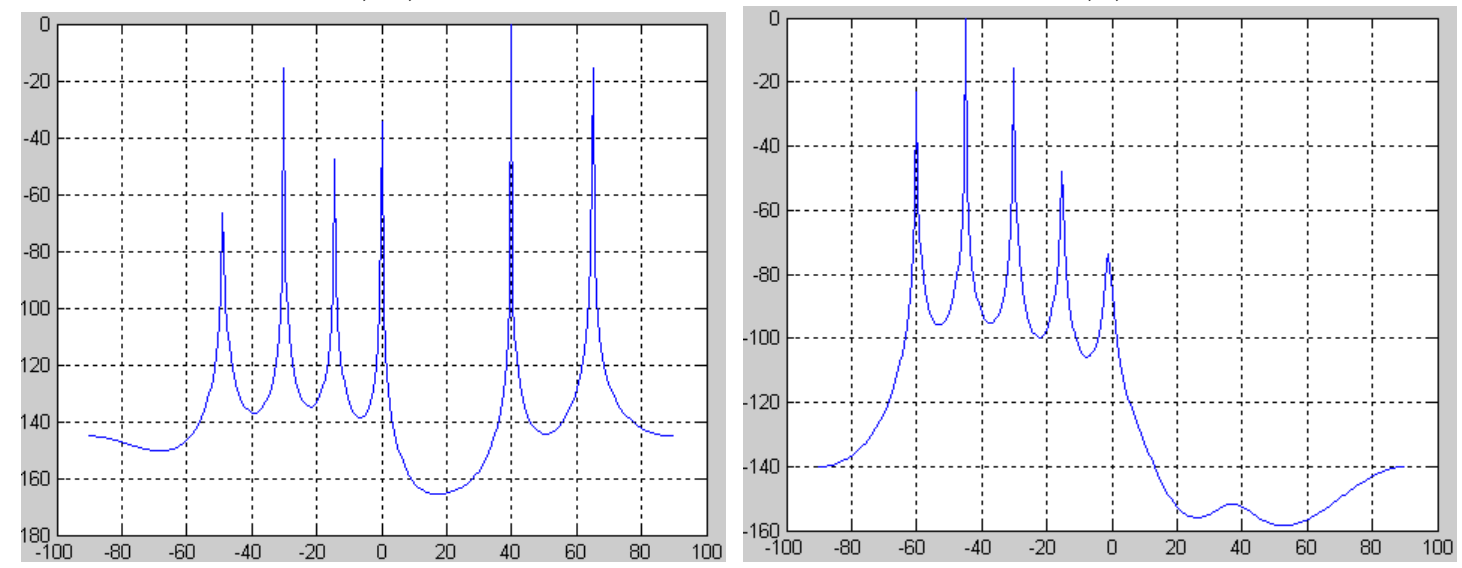

(c) incident direction:-30,40,65

(d) incident direction:-60,-45,-30

Fig.3. Estimation performance of different incident direction with beamforming MUSIC

Figure 3 shows that when incident angle difference is bigger, the beam space MUSIC algorithm can better get the DOA. Beam space matrix determines the performance, correctly estimate DOA requires adjacent beams under certain conditions.

\section{Spatial Smoothing MUSIC}

Spatial smoothing MUSIC divides uniform linear array of narrowband source into several overlapping subarray, averages the covariance matrix of each subarray to decorrelation. Forward spatial smoothing divides the $\mathrm{M}$ array into $\mathrm{p}$ subarray, each subarray has $\mathrm{m}$ arrays ${ }^{[4]}$, as shown in figure 4(a), covariance matrix is $R^{f}=\frac{1}{p} \sum_{i=1}^{p} R_{i}=A\left(\frac{1}{p} \sum_{i=1}^{p} D^{(i-1)} R_{s}\left(D^{(i-1)}\right)^{H} A^{H}+\sigma^{2} I\right.$ (4)

The subarray partition of backward spatial smoothing is shown in figure 4 (b), the model is similar to forward spatial smoothing, covariance matrix is

$$
R^{b}=\frac{1}{p} \sum_{i=1}^{p} R_{p-i+1}^{b}=A\left(\frac{1}{p} \sum_{i=1}^{p} D^{-(m+i-2)} R_{s}^{*} D^{(m+i-2)}\right) A^{H}+\sigma^{2} I
$$

The covariance matrix of forward/backward spatial smoothing MUSIC is the average of two before-mentioned matrices, namely

$$
R_{b f}=\frac{R^{f}+R^{b}}{2}{ }^{[5]} \text {. }
$$




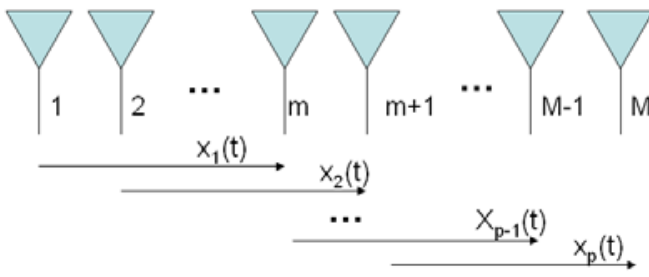

(a) forward spatial smoothing

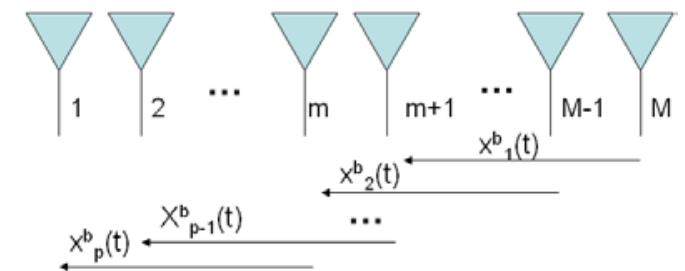

(b) backward spatial smoothing

Fig.4. Figure of subarray partition with spatial smoothing MUSIC

Experiment uses 16 array elements, 2 separate narrowband signal source, the center frequency is $1000 \mathrm{~Hz}$, signal-to-noise ratio is $10 \mathrm{~dB}$, incident to the linear array from 60 degrees and 30 degrees, the sampling number is 1024 , take half wavelength as array element spacing. Take forward spatial smoothing MUSIC and forward /backward spatial smoothing MUSIC for simulation, the results are shown in figure 5 .

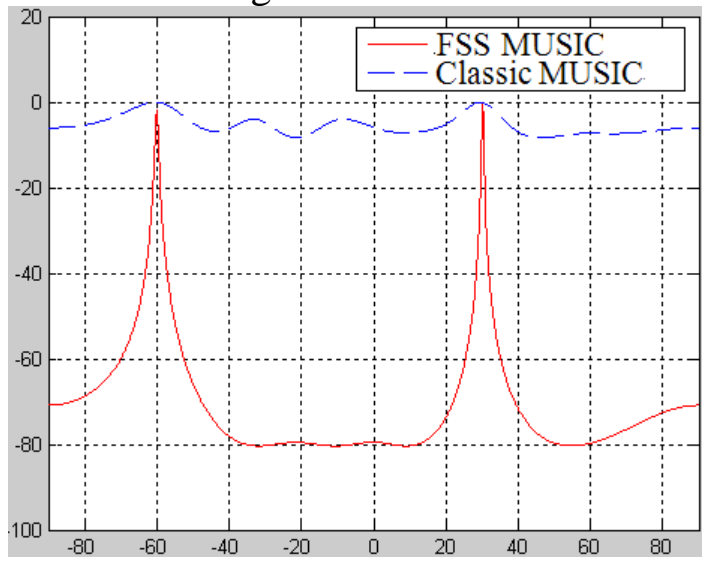

(a) forward spatial smoothing MUSIC

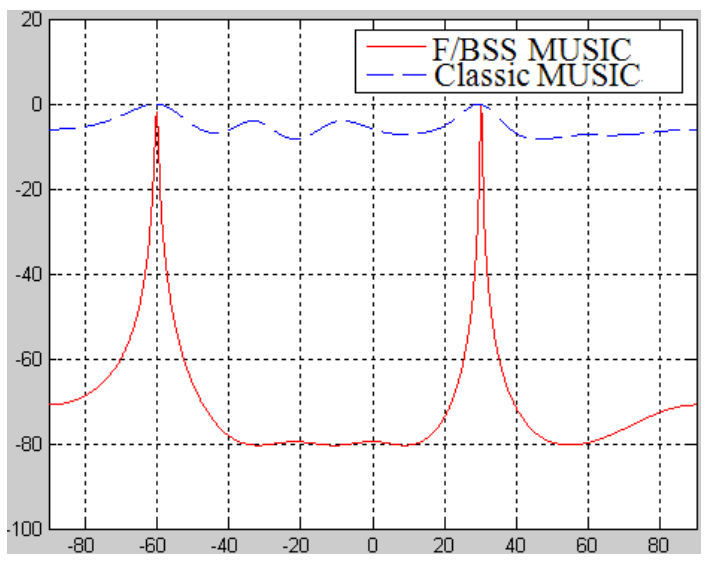

(b) forward/backward spatial smoothing MUSIC

Fig.5. Estimation performance of different spatial smoothing modes

Figure 5 shows that spatial smoothing technique can effectively estimate DOA of coherent signal with high precision by preprocessing the covariance of the input signal. Spatial smoothing reduce the front aperture and the number of array element, result in a decreased estimation performance of noncorrelated signals.

\section{Conclusion}

This paper analyzes the principle of three kinds of MUSIC algorithm, simulates and compares the DOA estimation performance with different references, the simulation results shows that Root-MUSIC algorithm has high precision under high SNR, beamforming MUSIC algorithm can accurately estimate DOA when incidence angle difference is large, the choice of beam space matrix determines the estimated performance, spatial smoothing MUSIC algorithm can effectively estimate the DOA of coherent signal with the cost of reducing antenna aperture, the choice should be made according to the situation and performance needs when ractical use.

\section{Acknowledgement}

In this paper, the research was sponsored by Philosophy and Social Science Foundation of Jiangsu Province (Project No. 2015SJD027) and Youth Fund Project of Chengxian college (Project No. y310001).

\section{References}

[1]Rao B D, Hari K V S. Performance analysis of Root-MUSIC. IEEE Trans. on ASSP, 1989 37(12) 1939-1949.

[2] A. Errikson, P. Stoica , T. Soderstorm. Markov-based eigenanalysis method for frequency 
estimation [J]. IEEE Trans. Signal Processiny, 1994 42(3) 586-594.

[3]P. N. Fletcher, P. Danwood . Beamforming for Circular and Semicircular Array An-tennas for Low-cost Wireless LAN Data Communications Systems. IEEE Proc-Microw Antennas Propagation, 1998 145(2)153-157.

[4]Pillai S.U, KWON B.H. Forward/Backward spatial smoothing technique for coherent signal identification, IEEE Trans. on ASSP,1989 37(1)8-15.

[5]T.J.Shan, M.Wax, T.Kailath. On spatial Smoothing for Direction-of-Arrival Estimation of Coherent Signals[J]. IEEE Transactions on Acoustic Speech and Signal Processing, 1985 33(4) 806-811. 